Research Paper

\title{
TNM Staging Matched-pair Comparison of Surgery After Neoadjuvant Chemoradiotherapy, Surgery Alone and Definitive Chemoradiotherapy for Thoracic Esophageal Squamous Cell Carcinoma
} \author{
Liu $^{1,2,3}$, Hong Yang1,2,4, Hui Liu ${ }^{1,2,3}$ \\ 1. State Key Laboratory of Oncology in South China; \\ 2. Guangdong Esophageal Cancer Research Institute, Guangzhou, Guangdong, P.R. China; \\ Department of Radiation Oncology: \\ Department of Thoracic Surgery; \\ Department of Endoscopy; \\ Department of Medical Oncology; \\ . Department of Clinical Statistics, Sun Yat-sen University Cancer Center, Guangzhou, Guangdong, P.R. China. \\ * contributed equally to this article.
}

ShiLiang Liu 1,2,3*, Bo Qiu 1,2,3*, GuangYu Luo ${ }^{1,2,5}$, Ying Liang1,2,6, YuZhen Zheng1,2,4, ZhaoLin Chen ${ }^{1,2,3}$, KongJia Luo ${ }^{1,2,4}$, Mian Xi1,2,3, Qing Liu1,2,7, YongHong $\mathrm{Hu}^{1,2,3}$, Qun $\mathrm{Li}^{1,2,3}$, JianHua Fu1,2,4, MengZhong

$\checkmark$ Corresponding author: Hui Liu, M.D., Ph.D., Department of Radiation Oncology, Sun Yat-sen University Cancer Center, 651 Dongfengdong Road, Guangzhou, Guangdong, P.R. China. Zip code: 510060. Tel.: 862087343031; Fax: 862087343492; E-mail address: liuhui@sysucc.org.cn.

(c) Ivyspring International Publisher. This is an open access article distributed under the terms of the Creative Commons Attribution (CC BY-NC) license (https://creativecommons.org/licenses/by-nc/4.0/). See http://ivyspring.com/terms for full terms and conditions.

Received: 2016.07.31; Accepted: 2016.11.16; Published: 2017.02.25

\begin{abstract}
Introduction: We used the TNM staging matched-pair approach to compare the efficacies of surgery after neoadjuvant chemoradiotherapy (NCT), surgery alone and definitive chemoradiotherapy (CCRT) in patients with localized advanced thoracic esophageal squamous cell carcinoma (ESCC). Methods: A total of 642 patients with ESCC from previous studies were studied. Patients whose treatment involved $\mathrm{NCT}+$ surgery and surgery alone were compared with patients receiving CCRT. Prospensity score matched-pair comparison based on pre-treatment TNM staging was developed to assess the efficacies of these treatment options. Results: Prospensity score matched-pair comparison to control for bias generated a cohort of 274 patients who were eligible for comparison. The 3 -year OS rate was $70.0 \%$ in the NCT + surgery group, compared to $51.7 \%$ in the surgery group $(p=0.000)$ and $61.9 \%$ in the CCRT group $(p=0.082)$. With the TNM staging matched-pair approach, the CCRT group had more upper thoracic ESCC patients (43/92, 46.7\%), while the surgery group had more lower thoracic ESCC patients (37/92, 40.2\%). The 3-year OS rates were comparable between the surgery alone group and CCRT group $(p=0.109)$. Conclusions: NCT plus surgery was superior in OS to surgery alone or CCRT. The 3-year OS rates were comparable between the surgery alone group and CCRT group with TNM staging matched-pair approach. Further investigation is warranted to confirm these findings.
\end{abstract}

Key words: Esophageal squamous cell carcinoma, neoadjuvant chemoradiotherapy, definitive chemoradiotherapy, surgery

\section{Introduction}

There are two main different histological types of esophageal cancer, adenocarcinoma and squamous cell carcinoma. Most clinical studies did not differentiate between these two types, but increasing evidence supports differences between esophageal adenocarcinoma and esophageal squamous cell carcinoma (ESCC) with respect to epidemiology, pathogenesis, tumor biology, and prognosis [1]. The most recent 2010 tumor, node, metastasis (TNM) staging system provides different stage groupings for 
ESCCs and adenocarcinomas of the esophagus in acknowledgement of these differences [2].

Surgery alone achieved poor long-term survival. Concurrent chemotherapy showed a radiosensitizing effect. These issues promoted the evaluation of preoperative chemoradiotherapy. Several randomized trials and meta-analyses supported better survival with neoadjuvant concurrent chemoradiation compared with surgery alone, and this multimodality approach is generally preferred for potentially resectable stage II/III thoracic cancer $[1,3,4]$. In the CROSS Trial, 363 patients diagnosed with potentially resectable esophageal or esophagogastric junction (EGJ) cancer (86 ESCC, 273 adenocarcinoma, and 4 other) were randomly assigned to preoperative chemoradiotherapy using weekly paclitaxel plus carboplatin and concurrent RT (41.4 Gy over five weeks) or surgery alone. The median overall survival (OS) was significantly better with preoperative chemoradiation (three-year survival rate $58 \%$ versus $44 \%$ ) at a median follow-up of 32 months [1].

Neoadjuvant chemoradiotherapy followed by surgery is superior, especially for thoracic esophageal adenocarcinoma, and the CROSS Trial demonstrated that histological type was not a prognostic factor for overall survival. Patients with ESCC benefited from neoadjuvant chemoradiotherapy the same as patients with adenocarcinoma [1]. However, the optimal treatment for locally advanced ESCC remains controversial in the real world, and clinical treatment includes surgery with or without preoperative treatment or a non-surgical approach using definitive chemoradiotherapy [5-10]. More ESCC patients had upper thoracic esophageal lesions and higher failures in cervical areas and the upper mediastinum after treatment. The cervical and superclavicular lymph nodes are difficult to remove using surgery, and the risk of anastomosis leak may be increased if this area was involved in the preoperative irradiation field.

Few published data have compared all the above-mentioned treatments for thoracic ESCC patients to address this question. Therefore, this study used the matched-pair approach as a less biased comparison of patients with similar clinical stages and other baseline characteristics to assist physicians in determining treatment options for localized advanced thoracic ESCC patients.

\section{Materials and Methods}

\section{Acquisition of clinical data}

The study scanned 642 consecutive patients who were diagnosed with locally advanced ESCC from previous studies $[11,21,22,23]$ between January 1, 2002, and June 30, 2012, at the Sun Yat-sen University
Cancer Center. A regional multidisciplinary team (MDT) fully discussed patient treatment options. Each patient underwent a physical examination, laboratory tests and electrocardiograms.,A medical history was acquired including concomitant medications, Eastern Cooperative Oncology Group (ECOG) performance status, and smoking/alcohol abusing history. Patient data included chest and upper abdomen computed tomography (CT) or positron emission tomography (PET) scans, endoscopic ultrasound (EUS) of the esophagus with biopsy, and bone scans. Tumor stage was classified using the TNM staging system proposed by the American Joint Committee on Cancer (6th edition). Patients satisfied the following inclusion criteria: 1) pathologically confirmed thoracic ESCC; 2) without previous cancer treatments; and 3) ECOG performance status $\leq 2$. Patients were excluded if their tumors were too stenotic to be examined by EUS or could not be processed on EUS examination for any other reasons. Patients with severe comorbid conditions and other active malignancies were excluded from the analysis. This study was a retrospective analysis of a prospectively assembled ESCC database. The Institutional Review Board of Sun Yat-Sen University Cancer Center approved the database and study design. Informed consent from all patients was obtained before treatment.

\section{Treatment}

Neoadjuvant chemoradiotherapy followed by surgery

There were two different concurrent chemotherapy regimens for neoadjuvant treatment: 1 . two cycles of cisplatin $\left(25 \mathrm{mg} / \mathrm{m}^{2}, \mathrm{~d} 1-4\right.$ and d 22-25) and NVB $\left(25 \mathrm{mg} / \mathrm{m}^{2}, \mathrm{~d} 1, \mathrm{~d} 8, \mathrm{~d} 22, \mathrm{~d} 29\right)$ concurrently with 40 Gy radiation, which was given in 20 fractions over 4 weeks; and 2 . weekly cisplatin $\left(25 \mathrm{mg} / \mathrm{m}^{2}, \mathrm{~d} 1\right.$, $\mathrm{d} 8, \mathrm{~d} 15$, and d 22) and docetaxel $\left(25 \mathrm{mg} / \mathrm{m}^{2}, \mathrm{~d} 1, \mathrm{~d} 8\right.$, d 15, and d 22) concurrently with 40 Gy radiation, which was administered in 20 fractions over 4 weeks. Radiotherapy was administered using a three-dimensional plan with a Varian Pinnacle radiotherapy treatment planning system. All patients underwent a physical examination, laboratory tests, electrocardiograms and lung function tests four weeks after neoadjuvant chemoradiotherapy (NCT). Patient evaluations also included chest and upper abdomen CT scans and EUS of the esophagus. Surgery was performed 6 weeks after neoadjuvant chemoradiotherapy. Surgery consisted of an initial right-sided posterolateral thoracotomy for esophagectomy, midline laparotomy for gastric tube mobilization, and a left-side cervical incision for anastomosis. A retrosternal or posterior mediastinal route was used for alimentary tract reconstruction. Lymph nodes (LNs) were resected and labeled by 
their specific location according to the guideline proposed by the Japanese Society for Esophageal Diseases [8]. Two-field lymphadenectomy was performed for patients with unfound cervical metastasis, including right/left laryngeal recurrent nerve, paraesophageal, subcarinal, superior phrenic, lower posterior mediastinal and abdominal lymph nodes.

\section{Surgery alone}

All patients were treated using the en bloc technique [6]. Briefly, the surgery consisted of an initial right-sided posterolateral thoracotomy for esophagectomy, midline laparotomy for gastric tube mobilization, and a left-side cervical incision for anastomosis. A retrosternal or posterior mediastinal route was used for alimentary tract reconstruction. LNs were resected and labeled by their specific location according to the guideline proposed by the Japanese Society for Esophageal Diseases. Our hospital routinely performed two-field lymphadenectomy for patients with unfound cervical metastasis, including right/left laryngeal recurrent nerve, paraesophageal, subcarinal, superior phrenic, lower posterior mediastinal and abdominal lymph nodes.

\section{Definitive chemoradiotherapy}

Patients were immobilized and simulated according to a standard protocol for esophageal carcinoma in our center [11]. Patients were immobilized with a vacuum in a supine position. CT-based simulation with $5 \mathrm{~mm}$ slice thickness was performed. Patients were scanned from the Axis (C2) to the second lumbar vertebra (L2) level. Briefly, the gross tumor volume (GTV) was the primary lesion diagnosed by endoscopy or CT scan. The regions of tumor that were discovered on endoscopy but not on CT were also included. A single radiologist (Dr. PeiQiangCai) reviewed all patient CT scans to minimize inter-observer variability. Lymph nodes that measured $\geq 10 \mathrm{~mm}$ in short axis, showed central necrosis or infiltrative margin were considered malignant [12]. The clinical target volume (CTV) of patients comprised subclinical areas. Two planning target volumes (PTVs) were defined. PTV1 covered the GTV with a $0.5-\mathrm{cm}$ margin, and PTV2 covered the CTV with a $0.5-\mathrm{cm}$ margin in all directions. Patients received a 3DCRT treatment plan that was calculated by the Philips Pinnacle planning system, and treatment was administered using a 6-MV linear accelerator. Patients received an IMRT treatment plan that was calculated by the Elekta Monaco planning system or Varian Eclipse planning system, and treatment was administered using a 6-MV linear accelerator. PTV1 was given a median dose of 60 Gy (range from 56-70 Gy) and PTV2 was given a median dose of $46 \mathrm{~Gy}$ (range from 40 to $54 \mathrm{~Gy}$ ). Organ constraints included a Dmax for the spinal cord $<46$ Gy, a mean dose for the lungs $<17$ Gy and a V20 for the lungs $<30 \%$.

There were two different concurrent chemotherapy regimens: 1 ) two cycles of cisplatin and 5-fluorouracil (5-FU), which consisted of $60 \mathrm{mg} / \mathrm{m}^{2}$ cisplatin administered on $\mathrm{d} 1$ and 29 and 300 $\mathrm{mg} / \mathrm{m}^{2} / 24 \mathrm{~h} 5-\mathrm{FU}$ administered on $\mathrm{d} 1$ to 3 and $\mathrm{d} 29$ to 31; and 2) cisplatin $25 \mathrm{mg} / \mathrm{m}^{2}$ and docetaxel 25 $\mathrm{mg} / \mathrm{m}^{2}$ weekly for $4-6$ weeks $[11,13]$.

\section{Follow-up and treatment response assessments}

The start of the follow-up period was defined as the initial date of local or systemic treatment. Chest and upper abdomen CTs were performed every 3 months and endoscopy every 6 months until the disease progressed. Bone scans were administered in patients with suspected bone metastasis. PET scans were performed in patients with suspected systemic progression. Treatment response, overall survival, local relapse and distant failure were recorded. OS was defined as the time from initial treatment (surgery or CRT) to death (or last known living contact), and disease-free survival (DFS) was defined as the time from initial treatment to disease failure. OS and DFS were used as measures of prognosis. Disease failure patterns were described as locoregional recurrence, distant recurrence, and combined failure. Locoregional recurrence was defined as recurrence at the anastomosis or within the area of the previous tumor resection or nodal clearance. Distant recurrence was defined as distant metastases to solid organs or recurrences in the pleura or peritoneal cavity. Combined failure was defined as simultaneous locoregional and distant recurrences.

\section{Statistical analysis}

Analysis of the general status (OS, PFS) of all 274 patients. OS was defined as the period from the initial date of treatment to the date of death from any cause or the last visit before December 31, 2014. PFS was calculated as the time from the initial date of treatment to the date of local or distant failures to the last visit before December 31, 2014, which was the date of last follow-up.

Means and standard deviations, frequencies and percentage were calculated to describe the data. A logistic regression model was used to generate propensity scores, including all potential confounding variables. Patient demographics were compared between three treatment groups by $\chi 2$ or MannWhitney $U$ test. The potential confounding variables 
were considered by the MDT and included age, ECOG performance status, EUS-defined clinical staging and CT-defined clinical staging. The Kaplan-Meier method was used to produce survival curves, and 3-year OS and DFS were determined. DFS and OS were compared using a two-sided log-rank test. IBM SPSS Statistics ver. 22 for Windows (IBM Corporation, Armonk, NY, USA) was used to perform all statistical analyses, and differences were considered significant for $\mathrm{p}<0.05$.

\section{Results}

\section{Patient characteristics}

A total of 274 of the 642 consecutive patients with locally advanced ESCC were eligible for inclusion. Based on the above-mentioned matching procedure and considering five matching criteria, 156 patients were excluded because of unavailable EUS examinations (Fig. 1). Table 1 shows the details of the 274 patients with respect to treatment modalities and matched tumor characteristics. Patient sex, age, clinical $\mathrm{T}$ stage, clinical $\mathrm{N}$ stage and clinical stage (AJCC $6^{\text {th }}$ edition) were well matched $(\mathrm{p}>0.5)$. More than $75 \%$ of patients in all three groups were diagnosed with stage III disease according to EUS and CT scans. There were $43 / 67(67.2 \%)$ patients with upper thoracic esophageal cancer in the CCRT group, and the NCT followed by surgery and surgery alone groups included more patients with primary tumors located at the middle and lower esophagus (Table 1).

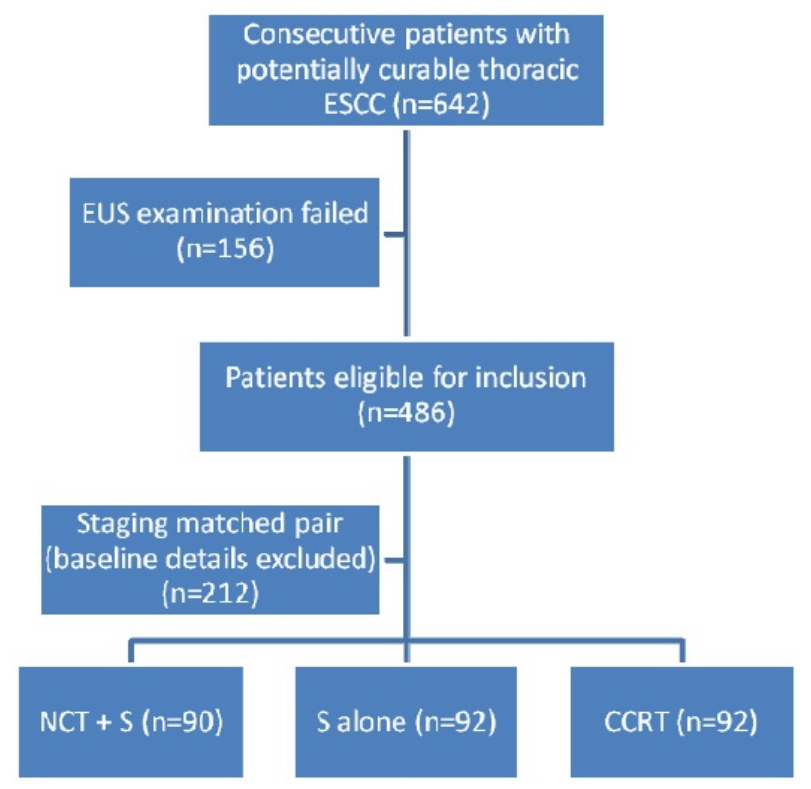

Fig. 1. Staging matched-pair analysis of thoracic ESCC patients who underwent radical treatments
Table 1. Clinical characteristics of thoracic ESCC patients who underwent radical treatments $(n=274)$

\begin{tabular}{|c|c|c|c|c|}
\hline Characteristics & $\begin{array}{l}\text { NCT+S }(n=90) \\
\text { No. }(\%)\end{array}$ & $\begin{array}{l}\text { Surgery } \\
(n=92) \text { No. }(\%)\end{array}$ & $\begin{array}{l}\text { CCRT }(n=92) \\
\text { No. }(\%)\end{array}$ & $\mathrm{p}$-value \\
\hline \multicolumn{5}{|l|}{ Sex } \\
\hline Male & $72(80.0 \%)$ & $70(76.1 \%)$ & $70(76.1 \%)$ & 0.862 \\
\hline Female & $18(20.0 \%)$ & $22(23.9 \%)$ & $22(23.9 \%)$ & \\
\hline $\begin{array}{l}\text { Age (year), median } \\
\text { (range) }\end{array}$ & $55(42-73)$ & $58(39-77)$ & $60(40-80)$ & 0.643 \\
\hline \multicolumn{5}{|l|}{ ECOG } \\
\hline $0-1$ & $88(97.8 \%)$ & $88(95.6 \%)$ & $85(92.4 \%)$ & 0.745 \\
\hline 2 & $2(2.2 \%)$ & $4(4.4 \%)$ & $7(7.6 \%)$ & \\
\hline \multicolumn{5}{|l|}{ cT stage (6 $6^{\text {th }}$ edition) } \\
\hline cT1 & $2(2.2 \%)$ & $2(2.2 \%)$ & $2(2.2 \%)$ & 0.884 \\
\hline cT2 & $18(20.0 \%)$ & $17(18.5 \%)$ & $17(18.5 \%)$ & \\
\hline cT3 & $65(72.2 \%)$ & $67(72.8 \%)$ & $67(72.8 \%)$ & \\
\hline cT4 & $5(5.6 \%)$ & $6(6.5 \%)$ & $6(6.5 \%)$ & \\
\hline \multicolumn{5}{|l|}{ cN stage (6 $6^{\text {th }}$ edition) } \\
\hline cN0 & $22(24.4 \%)$ & $25(27.2 \%)$ & $22(23.9 \%)$ & 0.872 \\
\hline cN1 & $68(75.6 \%)$ & $67(72.8 \%)$ & $70(76.1 \%)$ & \\
\hline \multicolumn{5}{|l|}{ cStage (6 $6^{\text {th }}$ edition) } \\
\hline Stage I & $2(2.2 \%)$ & $2(2.2 \%)$ & $2(2.2 \%)$ & 0.891 \\
\hline Stage II & $20(22.2 \%)$ & $21(22.8 \%)$ & $20(21.7 \%)$ & \\
\hline Stage III & $68(75.6 \%)$ & $69(75.0 \%)$ & $70(76.1 \%)$ & \\
\hline
\end{tabular}

\section{3-year OS by different treatments and tumor locations}

Patients demonstrated an estimated OS of 68.7 months in the NCT + surgery group, 52.7 months in the surgery alone group and 59.8 months in the CCRT group with a median follow-up of 48 months (range, 5-109 months) ( $\mathrm{p}=0.098$ using the log-rank test; HR, 1.188; $95 \%$ CI, 0.967 to 1.456) (Fig. 2A). The 3-year OS rate was $70.0 \%$ in the NCT + surgery group, compared with $51.7 \%$ in the surgery alone group ( $p=0.000$ using the log-rank test) and $61.9 \%$ in the CCRT group ( $p=0.082$ using the log-rank test). The 3 -year OS rates were comparable between the surgery alone group and CCRT group $(p=0.109$ using the log-rank test).

Sixty-seven of the 274 (24.5\%) ESCC patients had primary tumors located at the upper thoracic esophagus, $139 / 274(50.7 \%)$ had middle thoracic tumors and 68/274 (24.8\%) had lower thoracic tumors. The analyses revealed an estimated OS of 65.8 months in the upper thoracic group, 63.2 months in the middle thoracic group and 57.7 months in the lower thoracic group $(\mathrm{p}=0.446 \mathrm{using}$ the log-rank test; HR, 1.096; 95\% CI, 0.865 to 1.388) (Fig. 2B). The 3-year OS rates were $64.2 \%$ in the upper thoracic group, $53.9 \%$ in the middle thoracic group and $59.2 \%$ in the lower thoracic group. With the TNM staging matched-pair approach, the CCRT group had more Upper thoracic ESCC patients (43/92, 46.7\%), while the surgery group had more lower thoracic ESCC patients $(37 / 92,40.2 \%)$. 

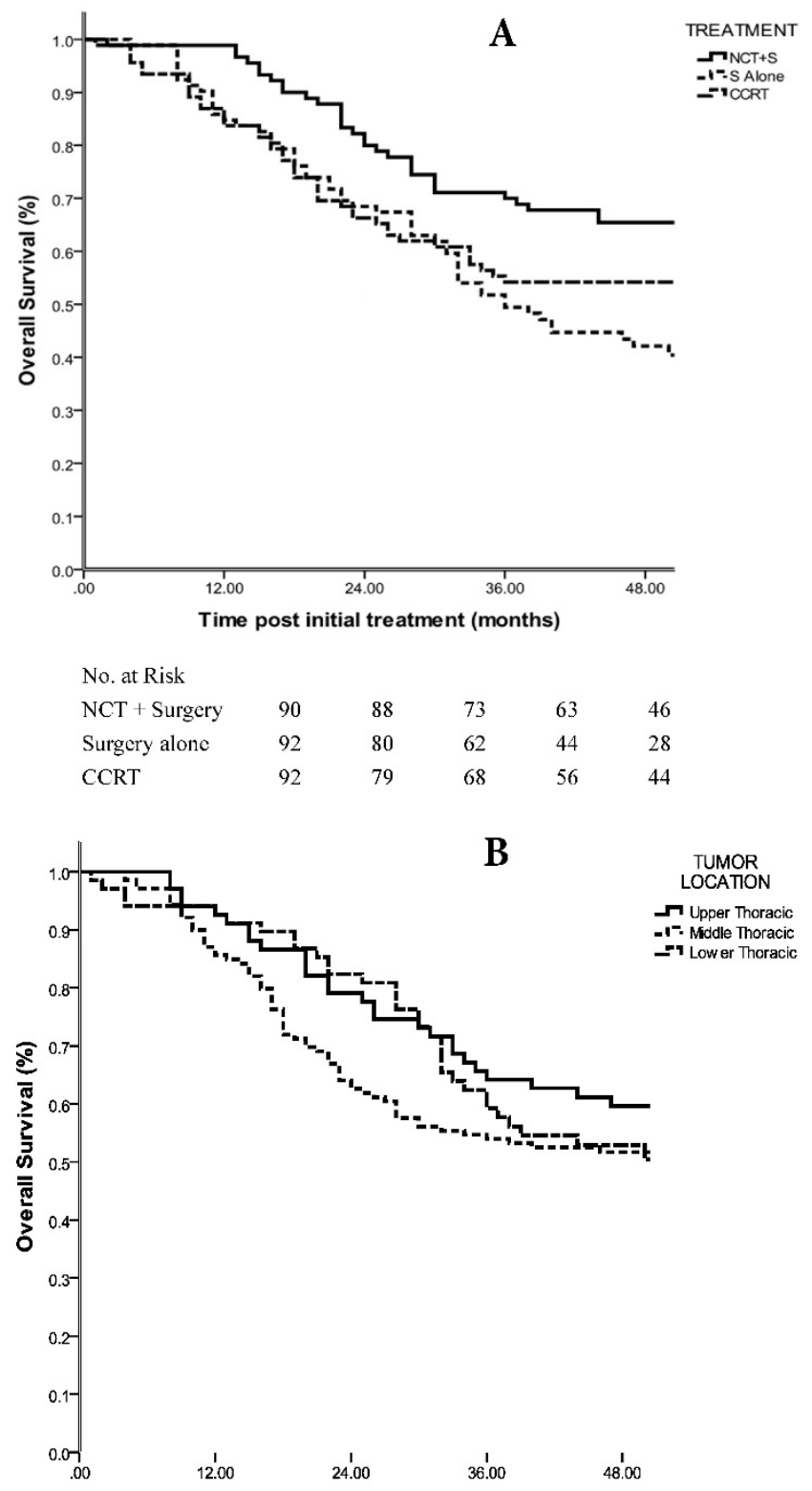

Time post initial treatment (months)

No. at Risk

$\begin{array}{llllll}\text { Upper Thoracic } & 67 & 62 & 52 & 43 & 36 \\ \text { Middle Thoracic } & 139 & 120 & 88 & 74 & 62 \\ \text { Lower Thoracic } & 68 & 63 & 55 & 39 & 24\end{array}$

Fig. 2. A. 3-year OS in patients with staging matched-pair ESCC treated by NCT + surgery, surgery alone and CCRT $(p=0.098)$. The difference between NCT + surgery group, and surgery alone group was significant $(p=0.000)$. The difference between NCT+surgery group and CCRT group was marginal significant $(p=0.082)$. The 3 -year OS rates were comparable between the surgery alone group and CCRT group $(p=0.109)$. B. 3-year OS in patients with staging matched-pair ESCC according to primary tumor location $(p=0.446)$.

\section{Toxic effects}

In NCT followed by surgery group, grade 3-4 hematologic toxicity was observed in 21 patients $(23.3 \%)$; 12 patients $(13.3 \%)$ developed pulmonary complications; 8 patients $(8.9 \%)$ had anastomotic leakage after surgery. In surgery alone group, 10 patients $(10.9 \%)$ had pulmonary complications and 9 patients $(9.8 \%)$ had anastomotic leakage. In CCRT group, 28 patients $(30.4 \%)$ developed grade 3-4 hematologic toxicity; 3 patients $(3.3 \%)$ developed febrile neutropenia; 4 patients $(4.3 \%)$ developed grade 3 pneumonitis.

\section{Failure patterns}

A total of 132 patients died during the observation period of the current study. The cause of death was disease progression in 124/132 (93.9\%) patients. Table 2 presents the failure patterns of all 274 patients who received the three different treatments. Local failure remained the main cause of death. However, NCT followed by surgery significantly decreased the incidence of loco-regional recurrence in thoracic ESCC $(p=0.026)$. The staging matched-pair method revealed that the incidence of distant metastases and multiple failures were comparable in all three groups.

Table 2. Treatments and patterns of failure of potentially curable $\operatorname{ESCC}(n=274)$

\begin{tabular}{|c|c|c|c|c|}
\hline Characteristic & $\begin{array}{l}\text { NCT + Surgery } \\
(n=90)\end{array}$ & $\begin{array}{l}\text { Surgery alone } \\
(n=92)\end{array}$ & $\begin{array}{l}\text { CCRT } \\
(\mathrm{n}=92)\end{array}$ & p-value \\
\hline Local recurrence (\%) & $\begin{array}{l}17(18.9 \%) \\
\text { (Cross Trial } \\
3.3 \% \text { ) }\end{array}$ & $\begin{array}{l}32(34.8 \%) \\
\text { (Cross Trial } \\
9.3 \%)\end{array}$ & $30(32.6 \%)$ & 0.026 \\
\hline $\begin{array}{l}\text { Distant metastasis } \\
(\%)\end{array}$ & $\begin{array}{l}14(15.6 \%) \\
\text { (Cross Trial } \\
24.2 \% \text { ) }\end{array}$ & $\begin{array}{l}18(19.6 \%) \\
\text { (Cross Trial } \\
22.0 \%)\end{array}$ & $11(12.0 \%)$ & 0.732 \\
\hline Multiple failures (\%) & $\begin{array}{l}8(8.9 \%) \\
\text { (Cross Trial } \\
10.8 \%)\end{array}$ & $\begin{array}{l}5(5.4 \%) \\
\text { (Cross Trial } \\
16.5 \%)\end{array}$ & $7(7.6 \%)$ & 0.874 \\
\hline $\begin{array}{l}\text { Total } \\
(\mathrm{n}=274)\end{array}$ & $39(43.3 \%)$ & $55(59.8 \%)$ & $48(52.2 \%)$ & 0.047 \\
\hline
\end{tabular}

Twenty-five patients in the NCT followed by surgery group developed local recurrences (17 local failures and 8 multiple failures). Ten of these patients had local recurrence at the upper mediastinum, 6 at the anastomosis, 11 in the supraclavicular area and 6 in the peritoneal area. Thirty-seven patients in the surgery alone group had local recurrences (32 local failures and 5 multiple failures). Twenty-one of these patients had local recurrence at the upper mediastinum, 16 at the anastomosis, 24 in the supraclavicular area and 10 in the peritoneal area. Thirty-seven patients in the CCRT group had local recurrences (30 local failures and 7 multiple failures). Twenty-eight of these patients had in-field local failure, and 9 had recurrent disease outside of the irradiation field.

\section{Discussion}

NCT followed by surgery, surgery and definitive CCRT are the main radical treatment options for localized thoracic ESCC nowadays [7]. Several 
randomized clinical trials directly compared the outcomes of esophageal cancer patients receiving esophagectomy alone with patients receiving NCT followed by surgery $[1,14,15]$. The results of the CROSS trial, including treatment efficacies and failure patterns, were published recently $[1,16]$. There was a significant estimated 5-year OS benefit of $13 \%$ in favor of the NCT + surgery group after a minimum follow-up of 24 months. There were only 46/366 $(12.6 \%)$ ESCC patients involved in this study, but the data analysis suggested that the benefit of NCT on survival was consistent across different histological subgroups [1]. However, there is no large-scale randomized trial that compared the treatment efficacies of NCT followed by surgery, surgery and definitive CCRT. One randomized clinical trial in the UK tried to compare NCT and surgery with definitive CCRT in localized ESCC, and the results demonstrated that this analysis was not feasible because of the low number of incident-eligible patients [17]. The results in the current study suggested that potentially curable ESCC patients who received NCT followed by surgery achieved a superior 3-year OS compared with those receiving surgery alone (estimated OS of 68.7 months in the $\mathrm{NCT}+$ surgery group, 52.7 months in the surgery group, $\mathrm{p}=0.000$ using the log-rank test), which is consistent with the previous published data from the CROSS trial. However, only a marginally significant $\mathrm{p}$-value $(\mathrm{p}=0.082$ using the log-rank test) was observed compared with the CCRT group (estimated OS of 68.7 months vs. 59.8 months). The staging matched pair analysis demonstrated comparable the 3-year OS rates between the surgery alone group and the CCRT group.

Karran et al. [18] reported a study of 727 esophageal cancer patients and used regression adjustment to create a cohort of 521 patients who were available for comparison (277 patients in the surgery group and 244 patients in the CCRT group). The median survival, and 2- and 5-year survival rates after surgery were 27 months, $53.8 \%$ and $31.0 \%$, respectively, compared with 28 months, $54.2 \%$ and $31.9 \%$ after CCRT $(p=0.918)$. The Bedenne et al.'s study [19] recruited ESCC patients who received $\mathrm{NCT}$, and patients who responded to NCT were randomized to undergo surgery or CCRT. The long-term treatment outcomes seemed comparable, but the study is limited because of the inclusion of only patients who responded to NCT. There was a lack of high-quality results comparing these three treatment options for locally advanced ESCC, but the current study demonstrated that NCT + surgery decreased the local recurrence of ESCC patients. The local recurrence rate in the NCT + surgery group was
$18.9 \%$, and it was $34.8 \%$ in the surgery alone group and $32.6 \%$ in the CCRT group ( $p=0.026)$. The benefit of local control using NCT + surgery might contribute to the better treatment outcome of ESCC patients compared with the other two groups.

NCCN guidelines recommend that ESCC patients with $\mathrm{T} 1 \mathrm{BN}+, \mathrm{T} 2-\mathrm{T} 4 \mathrm{aN} 0 \mathrm{~N}+$ receive $\mathrm{NCT}+$ surgery as radical treatment, but definitive CCRT should be administered to patients who are diagnosed with cervical primaries or who decline surgery. The current staging matched-pair study found that tumor location was an important issue for treatment selection, even in thoracic ESCC patients. Upper thoracic ESCC patients had a higher chance to receive definitive CCRT, while lower thoracic ESCC patients might be more likely to receive surgery-based treatment.

The AJCC $7^{\text {th }}$ edition staging system of esophageal cancer identifies clinical stage as the most important issue in making treatment decisions. There were few data on the impact of tumor location of thoracic ESCC of treatment outcomes, which may because most of the data came from surgical treatment. Previous studies suggested that upper thoracic ESCC patients had poorer long-term survival than middle thoracic and lower thoracic patients because of higher local recurrences. Our study demonstrated that upper thoracic ESCC patients in the NCT + surgery group and surgery alone group exhibited higher local recurrences at the cervical area, anastomosis and upper mediastinal area than patients who received definitive CCRT. A meta-analysis also demonstrated that the commonly metastatic areas of thoracic esophageal cancer were different according to the location of esophageal primary tumors. The metastatic rates of cervical, upper-mediastinal and mid-mediastinal nodes were $30.7 \%, 42.0 \%$ and $12.9 \%$, respectively for upper thoracic esophageal cancer. The rates of cervical, upper-mediastinal, mid-mediastinal and abdominal nodes in middle thoracic esophageal cancer were $16.8 \%, 21.1 \%, 28.1 \%$ and $21.4 \%$, respectively. The rates of cervical, upper-mediastinal, middle and lower mediastinal and abdominal nodes in lower thoracic esophageal cancer were $11.0 \%$, $10.5 \%, 19.6 \%, 23.0 \%$ and $39.9 \%$, respectively [20]. These findings suggest that cervical and upper-mediastinal nodes had higher risks to develop local recurrences in upper thoracic ESCC patients, and these areas should be covered by local therapy to prevent failures after radical treatment. Subclinical diseases in these areas are not easily treated using surgical methods, but these areas can be covered by radiotherapy without obvious side effects. Therefore, definitive CCRT could achieve promising long-term outcomes compared to surgical options. Subgroup 
analysis failed to demonstrate that tumor location was a significant risk factor for long-term treatment outcome in this entire cohort. The estimated OS rates in ESCC patients with upper thoracic, middle thoracic and lower thoracic disease were 65.8 months, 63.2 months and 57.7 months, respectively ( $p=0.446$ using $\log$ rank test). This result suggests that full discussions with MDT physicians before treatment may be useful for treatment decision-making and provide individualized treatment options for thoracic ESCC patients.

This retrospective study has some limitations, such as selection bias, small sample and short follow-up time. It was a nonrandomized, observational comparison of three treatment options and confounded by case mix. The use of staging matched-pairs in the analyses of overall and disease-free survival reduces some of these biases. However, randomized trials are warranted to validate our results.

The results of our TNM staging matched-pair approach for a less biased comparison with similar clinical stage and other baseline characteristics demonstrated that NCT + surgery remained a superior radical treatment option in thoracic ESCC patients. Upper thoracic ESCC patients had a higher chance to receive definitive CCRTwhile lower thoracic ESCC patients might be more suitable for surgery-based treatment. The 3-year OS rates were comparable between the surgery alone group and CCRT group with TNM staging matched-pair approach regardless of the location of tumor for thoracic ESCC patients. MDT discussion is important for making treatment decisions and achieving better treatment outcome. Further investigation is warranted to confirm these findings.

\section{Conflict of Interest} declare.

The authors have no conflicts of interest to

\section{Source of Funding}

This research was supported by 1 . funds from the University Cancer Foundation via the Sister Institution Network Fund at the University of Texas MD Anderson Cancer Center and supported in part by the National Institutes of Health through M. D. Anderson's Cancer Center Support Grant CA016672 as a portion of these studies were performed in the North Campus Flow Cytometry and Cellular Imaging Core (PI: Ronald A DePinho). 2. funds from the Scientific Research Foundation for the Returned Overseas Chinese Scholars, State Education Ministry. 3. National Nature Science Fund, Support Grant 81301932.

\section{Abbreviations}

NCT: neoadjuvant chemoradiotherapy; CCRT: definitive chemoradiotherapy; ESCC: esophageal squamous cell carcinoma; TNM: tumor, node, metastasis; EGJ: esophagogastric junction; OS: overall survival; MDT: multidisciplinary team; ECOG: Eastern Cooperative Oncology Group; CT: computed tomography; PET: positron emission tomography; EUS: endoscopic ultrasound; GTV: gross tumor volume; CTV: clinical target volume; PTV: planning target volume; 5-FU: 5-fluorouracil; DFS: disease-free survival.

\section{Competing Interests}

The authors have declared that no competing interest exists.

\section{References}

1. Van Hagen P, Hulshof MC, Van Lanschot JJ, et al. Preoperative chemoradiotherapy for esophageal or junctional cancer. N Engl J Med. 2012; 366: 2074-2084.

2. Talsma K, Van Hagen P, Grotenhuis BA et al. Comparison of the 6th and 7th Editions of the UICC-AJCC TNM Classification for Esophageal Cancer. Ann Surg Oncol. 2012; 19: 2142.

3. Sjoquist KM, Burmeister BH, Smithers BM, et al. Survival after neoadjuvant chemotherapy or chemoradiotherapy for resectable oesophageal carcinoma: an updated meta-analysis. Lancet Oncol. 2011; 12:681-692.

4. Ronellenfitsch U , Schwarzbach M, Hofheinz R, et al. Preoperative chemo(radio)therapy versus primary surgery for gastroesophageal adenocarcinoma: systematic review with meta-analysis combining individual patient and aggregate data. Eur J Cancer. 2013; 49:3149-3158.

5. Allum WH, Stenning SP, Bancewicz J, et al. Long-term results of a randomized trial of surgery with or without preoperative chemotherapy in esophageal cancer. J Clin Oncol. 2009; 27:5062-5067.

6. Stahl $\mathrm{M}$. Is there any role for surgery in the multidisciplinary treatment of esophageal cancer? Ann Oncol. 2010;21 (Suppl 7):vii283-vii285.

7. Stahl M, Mariette C, Haustermans K, et al. Oesophageal cancer: ESMO Clinical Practice Guidelines for diagnosis, treatment and follow-up. Ann Oncol. 2013; 24 Suppl 6:vi51-6

8. Japanese Society for Esophageal Diseases. Clinicopathological aspects. In: Guidelines for clinical and pathologic studies on carcinoma of the esophagus. Tokyo: Kanehara \& Co Ltd. 1999:1-34.

9. Gwynne S, Hurt C, Crosby T, et al. Definitive chemoradiation for oesophageal cancer-a standard of care in patients with non-metastatic oesophageal cancer. Clin Oncol (R Coll Radiol). 2011; 23(3):182-8.

10. Yamamoto $S$, Ishihara $R$, Iishi $H$. Comparison between definitive chemoradiotherapy and esophagectomy in patients with clinical stage I esophageal squamous cell carcinoma. Am J Gastroenterol. 2011; 106(6):1048-54.

11. Li QQ, Liu MZ, Hu YH, et al. Definitive concomitant chemoradiotherapy with docetaxel and cisplatin in squamous esopheageal carcinoma. Dis Esophagus. 2010; 23(3):253-9.

12. $\mathrm{Wu} \mathrm{KL}$, Chen GY, $\mathrm{Xu} \mathrm{ZY,} \mathrm{et} \mathrm{al.} \mathrm{Three-dimensional} \mathrm{conformal} \mathrm{radiation}$ therapy for squamous cell carcinoma of the esophagus: a prospective phase I/II study. Radiother Oncol. 2009; 93(3):454-7.

13. Day FL, Leong T, Michael M, et al. Phase I trial of docetaxel, cisplatin and concurrent radical radiotherapy in locally advanced oesophageal cancer. Br J Cancer. 2011; 104(2):265-71.

14. Urba SG, Orringer $\mathrm{MB}$, Strawderman $\mathrm{M}$, et al Randomized trial of preoperative chemoradiation versus surgery alone in patients with locoregional esophageal carcinoma. J Clin Oncol. 2001; 19(2):305-13.

15. Burmeister $\mathrm{BH}$, Smithers BM, Danham JW, et al. Trans-Tasman Radiation Oncology Group; Australasian Gastro-Intestinal Trials Group (2005) Surgery alone versus chemoradiotherapy followed by surgery for resectable cancer of the oesophagus: A randomized controlled phase III trial. Lancet Oncol. 2005; 6(9):659-68.

16. Oppedijk V, van der Gaast A, van Lanschot JJ, et al. Patterns of recurrence after surgery alone versus preoperative chemoradiotherapy and surgery in the CROSS trials. J Clin Oncol. 2014; 32(5):385-91.

17. Blazeby JM, Strong S, Brookes ST, et al. Feasibility RCT of definitive chemoradiotherapy or chemotherapy and surgery for oesophageal squamous cell cancer. Br J Cancer. 2014 Jul 15; 111(2):234-40. 
18. Karran A., Blake P., Lewis G., et al. Propensity score analysis of oesophageal cancer treatment with surgery or definitive chemoradiotherapy. Br J Surg. 2014 Apr; 101(5):502-10.

19. Bedenne L, Michel P, Bouché O, et al. Chemoradiation followed by surgery compared with chemoradiation alone in squamous cancer of the esophagus: FFCD 9102. J Clin Oncol. 2007 Apr 1; 25(10):1160-8.

20. Ding X, Zhang J, Li H. et al. A meta-analysis of lymph node metastasis rate for patients with thoracic oesophageal cancer and its implication in delineation of clinical target volume for radiation therapy. Br J Radiol. 2012; 85(1019):e1110-9.

21. Liu SL, Yang $\mathrm{H}$, Zhang $\mathrm{P}$, et al. Neoadjuvant chemoradiotherapy with cisplatin plus vinorelbine versus cisplatin plus fluorouracil for esophageal squamous cell carcinoma: A matched case-control study. Radiother Oncol. 2015; 116(2):262-8.

22. Qiu B, Wang D, Yang H, et al. Combined Modalities of Magnetic Resonance Imaging, Endoscopy, and Computed Tomography in Evaluation of Tumor Response to Definitive Chemoradiation Therapy in Esophageal Squamous Cell Carcinoma. Radiother Oncol. 2016; [Epub ahead of print].

23. Liu S, Anfossi S, Qiu B, et al. Prognostic Factors for Locoregional Recurrence in Patients with Thoracic Esophageal Squamous Cell Carcinoma Treated with Radical Two-Field Lymph Node Dissection: Results from Long-Term Follow-Up. Ann Surg Oncol. 2016; [Epub ahead of print] 